\title{
On the Extension of the Baryonic Tully-Fisher Relation to Galaxy Clusters and Super Massive-Cosmic Systems
}

\author{
Barbaro Quintero-Leyva \\ Miami, Florida, USA \\ Email: Doserate2002@yahoo.com
}

How to cite this paper: Quintero-Leyva, B. (2017) On the Extension of the Baryonic Tully-Fisher Relation to Galaxy Clusters and Super Massive-Cosmic Systems. Open Access Library Journal, 4: e3686. https://doi.org/10.4236/oalib.1103686

Received: May 23, 2017

Accepted: June 11, 2017

Published: June 14, 2017

Copyright (c) 2017 by author and Open Access Library Inc.

This work is licensed under the Creative Commons Attribution International License (CC BY 4.0).

http://creativecommons.org/licenses/by/4.0/

\section{c) (i) Open Access}

\begin{abstract}
The Baryonic Tully-Fisher relation was extended to clusters hypothesizing that $a_{0}$, the characteristic acceleration of the Modified Newtonian Dynamics (MOND), depends on the mass of the system. Circular speeds were calculated for systems with mass up to $10^{21} \mathrm{M}_{\odot}$. The relativistic impact on super massive systems was considered using the extended Newtonian theory for gravitational bound systems. The impact was an increase of the circular velocity for systems with mass beyond $\sim 10^{19} \mathrm{M}_{\odot}$.
\end{abstract}

\section{Subject Areas}

Mechanics, Modern Physics, Numerical Mathematics

\section{Keywords}

Dark Matter, Dark Energy, Galaxies: Kinematics and Dynamics, Clusters, Cosmology

\section{Introduction}

A historical and technical background to the cosmological problem currently known as dark matter (or missing mass) was presented in [1] where references to works and ideas dating back at least to Newton's time were made.

In 1933 ref. [2] concerning the red shift of extragalactic nebulae reported that the magnitude of the measured velocity dispersion of the Coma cluster implies much more mass than the mass estimated from the cluster luminosity-mass because the calculation of the velocity dispersion from the luminosity mass using the virial theorem yielded much lower velocity dispersion. He therefore inferred, as one possibility, the presence of dark matter in greater amount than the lu- 
minous mass to explain the discrepancy. In 1937 ref. [3] goes into more detail and refinement of the findings of 1933.

In the1970s the need for undetected additional mass in the outer part and well beyond the optical edge of the galaxy where the rotation curves showed an effective flat profile, started emerging ([1] and references there in),

Ref. [4] established a relation between the stellar luminosity (L) of the galaxies and the global profile width $\left(\Delta \mathrm{V}(0)\right.$ in $\left.\mathrm{km} \mathrm{s}^{-1}\right)$, for the Virgo cluster, they anticipated $L \propto \Delta \mathrm{V}^{3.1}$. The subject of that paper however was not the missing mass problem but the determination of distances to galaxies.

To explain the dark matter problem many scientists were in the search for a particle or object that does not interact electromagnetically while it does gravitationally. Two efforts in this direction are: WIMP (weakly interacting massive particle: non baryonic, it has not been detected) and MACHO (massive astronomical compact halo objects: baryonic, according to [1], it does not account for the total needed dark matter in, for example, the Milky Way).

A modification of the Newtonian dynamic (MOND) was proposed in 1983 [5] as an alternative to the search for WIMP and MACHO, in such a way that for very low acceleration regime an asymptotically flat rotation curve is obtained well outside the optical edge of spiral galaxies and for very high acceleration the Newtonian dynamics is recovered. MOND assumes the existence of a characteristic acceleration about $10^{-10} \mathrm{~ms}^{-2}$ which is about $1 / 6$ of the current acceleration of the universe. MOND predicted a Tully-Fisher relation (TFR) of the form $M \propto \mathrm{V}^{4}$ where $M$ and $\mathrm{V}$ are the galaxy mass and the asymptotically flat circular velocity respectively.

In 1999 [6], it was found that by adding the HI component to the stellar mass, the TFR is recovered in the DDO 154 and NGC 2115 (gas rich) galaxies. In 2000 [7] the TFR was extended over 5 decades in stellar mass and it was found that the stellar TFR can hold for large dwarf galaxies (previously believed not to follow the TFR) if the mass of the gas is added to the stellar mass (establishing therefore a Baryonic TFR), consistent with the finding in [6], confirming in this way the MOND prediction $M \propto \mathrm{V}^{4}$ very closely.

In 2006 ref. [8] concerning the merge of two clusters (known as the Bullet cluster or1E0657-558) found that the mass of the system does not follow the distribution of baryons (based on gravitational lensing and hot $\mathrm{x}$-ray emission measurements). However work in the MOND direction continued, for example, a MOND model for the bullet cluster was proposed in [9].

Alternatives to MOND phenomenology (in addition to the dark matter hypothesis) can be found in the literature: e.g. In 1988 the magnetic stress on the outer gas of disk galaxies was found to yield rotation velocities well above to what is expected based on the visible matter [10]; In 2005 a modified acceleration law based on the Einstein gravity coupled to a massive skew symmetric field (Metric Skew Tensor Gravity) was used to explain the rotation curve of galaxies. MSTG assumes a space and mass dependence of the Newton's gravitational constant and a characteristic radius and mass [11]. In 2011 a generalization of the New- 
ton's gravitational theory based on the work of Heaviside and Sciama (implementing the Mach principle) was proposed resulting in a dynamic model for a galaxy without dark matter [12]; In 2012 It was proposed that the dark matter problem is the result of an inertial effect due to a postulated cosmic rotation [13]; In 2012 the inertial mass is modified assuming that the Unruh radiation is subject to a Hubble scale-Casimir effect dubbed Quantized Inertia [14]. QI predicts the BTF relation with a characteristic acceleration which is very close to the universe acceleration being therefore about six times the MOND's one.

It will be seen in the next section that QI systematically overestimates the cluster circular speed while MOND systematically and very significantly under estimates it, however MOND estimates the circular speed of the disk galaxies much better than what QI does.

The purpose of this work is to extend the Baryonic Tully-Fisher relation (in the MOND context) to galaxy clusters and super massive-cosmic systems. To fulfill this purpose it is hypothesized that the MOND's characteristic acceleration is not a constant and that a mass dependence could be attributed to it, extending in this way the BTF relation to galaxy clusters. The extension of the BTF relation to very massive systems (where the speed is a significant fraction of the speed of light in vacuum) is made using the extended Newtonian theory for gravitational bound systems [15] which successfully accounts for the perihelion precession of the planets of the solar system.

\section{Extension of the BTF Relation to Galaxy Clusters}

\subsection{MOND's BTF for Galaxies}

MOND described as a modification of the Newton's $2^{\text {nd }}$ law can be written as [5]:

$$
F_{g}=\operatorname{ma\mu }\left(a / a_{0}\right)
$$

where

$F_{g}$ : Newtonian gravitational force acting on a test particle of mass $m$

$a$ : Newtonian acceleration of $m$

$\mu\left(a / a_{0}\right)$ : An interpolating function that is equal to $a / a_{0}$ for $a \ll a_{0}$ and is equal to 1 when $a \gg a_{0}$

$a_{0}:$ MOND characteristic acceleration.

If the simple interpolating function [16], $\mu(x)=\frac{x}{1+x}$, is used and the establishment of an acceleration given by $\frac{\mathrm{v}^{2}}{r}$ is assumed, Equation (1) becomes

$$
\mathrm{v}^{4}-F(r) r \mathrm{v}^{2}-F(r) r^{2} a_{0}=0
$$

$\mathrm{v}$ : Circular speed

$F(r)$ : Force per unit of test particle mass

$r$ : Distance from the center of the system to the location of the test particle.

If the impact of the geometry on the force field and the effect of the mass beyond the location of the test particle are neglected then $F(r)=\frac{G M(r)}{r^{2}}$ and 
Equation (2) can be written as

$$
\mathrm{v}^{4}-G \mathrm{M}(r) \frac{\mathrm{v}^{2}}{r}-G \mathrm{M}(r) a_{0}=0
$$

$G$ : Newton gravitational constant.

Equation (3) for very small acceleration becomes

$$
\mathrm{v}^{4}=G M a_{0}
$$

\subsection{QI BTF Relation}

The BTF relation derived in [14] for the edge of the galaxy is:

$$
\mathrm{v}^{4}=G M a_{0}, \quad a_{0}=2 c^{2} / \Theta=6.7 \times 10^{-10} \mathrm{~m} \cdot \mathrm{s}^{-2}
$$

where

$c$ : Speed of light in vacuum

$\Theta$ : Hubble diameter

\subsection{Extension of the BTF Relation to Clusters of Galaxies}

Independently of the physical background (MOND, QI, etc.) behind the effective flatness of the rotational curve after the optical edge of the galaxies, it is has been experimentally well established in disk galaxies the existence of a BTF relation of the form of Equation (4).

Even though Equation (4) works remarkable well for many disk galaxies, it however underestimates the circular speed for clusters as can be seen from Table 1 (MOND using $a_{0}=1.16 \times 10^{-10} \mathrm{~m} \cdot \mathrm{s}^{-2}$ ). Note in that table that when using $a_{0}=6.7 \times 10^{-10} \mathrm{~m} \cdot \mathrm{s}^{-2}(\mathrm{QI})$, the results match better the clusters than the MOND does, but MOND performs better for disk/gas galaxies. These observations were previously noted in [14] for this binned experimental data set.

In Figure 1 (see Table 1 also) is plotted the values of $a_{0}$ vs. $M$ calculated per Equation (4) using the binned experimental values of masses and velocities shown in Table 1. It can be seen a strong correlation for the clusters. Based on that and assuming that the distances to the cosmic structures in question were accurately determined, it is hypothesized that $a_{0}$ is not really a constant and that it can conveniently be represented (without considering the binned data for the dwarf galaxies) with a function of the form

$$
a_{0}(M)=\frac{c H_{0}}{k}(1+(k-1) \eta(M)), k>1
$$

where,

$\eta(M)$ : Monotonously increasing function of $M$ with the constraints: $\eta(0)=0, \eta\left(M_{U}\right)=1$.

$M_{U}:$ Mass of the universe

Of course, $\eta(M)$ should represent the experimental data reasonable well.

If it is found out that $a_{0}(0)=0$ then Equation (5) should be written as $a_{0}(M)=c H_{0} \eta(M)$.

Because the exact shape of $\eta(M)$ is not yet known $a_{0}(M)$ is determined 
Table 1. Circular velocity vs. baryonic mass $\left(\mathrm{M}_{\mathrm{b}}\right): \mathrm{V}_{\mathrm{c}}$ : Binned experimental value; QI (Quantized Inertia); HYB (mass dependent $a_{0}$ ). The binned data set (columns 1 - 3) was taken from [17].

\begin{tabular}{|c|c|c|c|c|c|c|c|c|c|}
\hline System & $\begin{array}{c}\mathrm{Vc} \\
\log 10\end{array}$ & $\begin{array}{c}\mathrm{Mb} \\
\log 10\end{array}$ & $\begin{array}{c}\mathrm{Vc} \\
(\mathrm{km} / \mathrm{s})\end{array}$ & $\begin{array}{c}\mathrm{Mb} \\
\text { (Msun) }\end{array}$ & $\begin{array}{c}a_{0}(\text { Equation }(4)) \\
\left(\mathrm{m} / \mathrm{s}^{2}\right)\end{array}$ & $\begin{array}{l}\text { MOND } \\
(\mathrm{km} / \mathrm{s})\end{array}$ & $\begin{array}{c}\text { QI } \\
(\mathrm{km} / \mathrm{s})\end{array}$ & $\begin{array}{c}a_{0}(\text { Equation }(6)) \\
\left(\mathrm{m} / \mathrm{s}^{2}\right)\end{array}$ & $\begin{array}{l}\text { HYB } \\
(\mathrm{km} / \mathrm{s})\end{array}$ \\
\hline cluster & 3.22 & 14.00 & 1659.59 & $1.00 \mathrm{E}+14$ & $5.72 \mathrm{E}-10$ & 1114.00 & 1726.67 & $5.71 \mathrm{E}-10$ & 1659.01 \\
\hline cluster & 3.10 & 13.57 & 1258.93 & $3.72 \mathrm{E}+13$ & $5.10 \mathrm{E}-10$ & 869.73 & 1348.06 & $5.11 \mathrm{E}-10$ & 1259.98 \\
\hline Cluster & 2.96 & 13.14 & 912.01 & $1.38 \mathrm{E}+13$ & $3.78 \mathrm{E}-10$ & 679.02 & 1052.47 & $3.63 \mathrm{E}-10$ & 902.66 \\
\hline Cluster & 2.84 & 12.76 & 691.83 & $5.75 \mathrm{E}+12$ & $3.00 \mathrm{E}-10$ & 545.61 & 845.69 & $2.81 \mathrm{E}-10$ & 680.43 \\
\hline Cluster & 2.71 & 12.46 & 512.86 & $2.88 \mathrm{E}+12$ & $1.81 \mathrm{E}-10$ & 459.08 & 711.56 & $2.47 \mathrm{E}-10$ & 554.57 \\
\hline Spiral & 2.40 & 11.32 & 251.19 & $2.09 \mathrm{E}+11$ & $1.44 \mathrm{E}-10$ & 238.17 & 369.16 & $2.14 \mathrm{E}-10$ & 277.43 \\
\hline Spiral & 2.32 & 10.99 & 208.93 & $9.77 \mathrm{E}+10$ & $1.47 \mathrm{E}-10$ & 196.96 & 305.29 & $2.12 \mathrm{E}-10$ & 229.04 \\
\hline Spiral & 2.23 & 10.63 & 169.82 & $4.27 \mathrm{E}+10$ & $1.47 \mathrm{E}-10$ & 160.10 & 248.15 & $2.12 \mathrm{E}-10$ & 186.01 \\
\hline Spiral & 2.15 & 10.26 & 141.25 & $1.82 \mathrm{E}+10$ & $1.65 \mathrm{E}-10$ & 129.39 & 200.54 & $2.11 \mathrm{E}-10$ & 150.27 \\
\hline Spiral & 2.08 & 10.00 & 120.23 & $1.00 \mathrm{E}+10$ & $1.57 \mathrm{E}-10$ & 111.40 & 172.67 & $2.11 \mathrm{E}-10$ & 129.37 \\
\hline Gas Disk & 2.07 & 9.85 & 117.49 & $7.08 \mathrm{E}+09$ & $2.03 \mathrm{E}-10$ & 102.18 & 158.38 & $2.11 \mathrm{E}-10$ & 118.66 \\
\hline Spiral & 2.03 & 9.79 & 107.15 & $6.17 \mathrm{E}+09$ & $1.61 \mathrm{E}-10$ & 98.72 & 153.01 & $2.11 \mathrm{E}-10$ & 114.63 \\
\hline Spiral & 1.92 & 9.31 & 83.18 & $2.04 \mathrm{E}+09$ & $1.77 \mathrm{E}-10$ & 74.88 & 116.07 & $2.11 \mathrm{E}-10$ & 86.95 \\
\hline Gas Disk & 1.88 & 9.21 & 75.86 & $1.62 \mathrm{E}+09$ & $1.54 \mathrm{E}-10$ & 70.69 & 109.57 & $2.11 \mathrm{E}-10$ & 82.09 \\
\hline Gas Disk & 1.78 & 8.62 & 60.26 & $4.17 \mathrm{E}+08$ & $2.38 \mathrm{E}-10$ & 50.34 & 78.02 & $2.11 \mathrm{E}-10$ & 58.45 \\
\hline Gas Disk & 1.65 & 8.24 & 44.67 & $1.74 \mathrm{E}+08$ & $1.73 \mathrm{E}-10$ & 40.45 & 62.69 & $2.11 \mathrm{E}-10$ & 46.96 \\
\hline Gas Disk & 1.37 & 7.28 & 23.44 & $1.91 \mathrm{E}+07$ & $1.19 \mathrm{E}-10$ & 23.27 & 36.08 & $2.11 \mathrm{E}-10$ & 27.02 \\
\hline Dwarf & 1.29 & 6.67 & 19.50 & $4.68 \mathrm{E}+06$ & $2.33 \mathrm{E}-10$ & 16.38 & 25.39 & $2.11 \mathrm{E}-10$ & 19.02 \\
\hline Dwarf & 1.16 & 5.60 & 14.45 & $3.98 \mathrm{E}+05$ & $8.26 \mathrm{E}-10$ & 8.85 & 13.72 & $2.11 \mathrm{E}-10$ & 10.27 \\
\hline Dwarf & 0.94 & 3.81 & 8.71 & $6.46 \mathrm{E}+03$ & $6.72 \mathrm{E}-09$ & 3.16 & 4.89 & $2.11 \mathrm{E}-10$ & 3.67 \\
\hline
\end{tabular}

Note in Table 1 that MOND underestimates also the binned experimental values for the dwarf galaxies. In this work these galaxies will not be considered (as input to any fitting process) because, apart from being accompanied with larger uncertainties, they include the local group dwarf galaxies that some of them appear not to be isolated and also because of the potential impact of the hypothesis formulated by [18] on the binned data.

by fitting the values of $a_{0}$ vs. $M$ of Table 1 . There are many ways of fitting the data with the constraints of equation 5 (e.g. spline fitting/interpolation). In this work a $3^{\text {rd }}$ degree polynomial with a preset intercept (to avoid significant artificial curvature between the last two points of Figure 1) without considering the constraint, $a_{0}\left(M_{U}\right)=c H_{0}$, was used. The resulting equation is

$$
a_{0}(M)=6.10 \times 10^{-52} M^{3}-1.55 \times 10^{-37} M^{2}+1.30 \times 10^{-23} M+2.11 \times 10^{-10}
$$

where, $M$ and $a_{0}(M)$ are expressed in solar masses and $\mathrm{m} / \mathrm{s}^{2}$ respectively. It is noted that [17] used instead a broken power law ( $\mathrm{v}$ as a function of $M$ ) to generalize the BTF relation for clusters.

It is curious that some polynomials ( $M$ as a function of $\mathrm{v}$ ) fitted to the binned data represent fairly well the data (better than a power law) with no significant susceptibility to produce artificial curvature (except for speeds smaller than about $160 \mathrm{~km} / \mathrm{s}$ where a power law fits better), 


$$
\text { e.g. } M=1.506 \times 10^{1} \mathrm{v}^{4}-1.333 \times 10^{4} \mathrm{v}^{3}+1.847 \times 10^{7} \mathrm{v}^{2}-2.522 \times 10^{9} \mathrm{v}
$$

However a polynomial fit of $\mathrm{v}$ as a function of $M$ yields pronounced artificial curvatures in the cluster region.

The extrapolation to more massive systems up to $M=M_{U}$ should be made, if needed, with another function because Equation (6) does not fulfill the condition $a_{0}\left(M_{U}\right)=c H_{0}$. That extrapolation, when not highly accurate results are demanded, could approximately be considered linear from the last point of Figure 1 because $a_{0}$ changes only in that interval about $1 \times 10^{-10} \mathrm{~ms}^{-2}$ over many orders of magnitude in $M$ and because $a_{0}$ seems to be leveling off.

The results labeled HYB in Table 1 correspond to the use of Equation (6) in Equation (4). It can be seen that HYB is in significant better agreement, in almost all points, with the binned measured velocities than both MOND (it underestimates the binned velocities, more significantly the clusters) and QI (it overestimates the binned velocities)

\section{BTF Extension to Super Massive Cosmic Structures}

When the mass of the cosmic structure is very large, the circular velocity is a significant percent of the speed of light and therefore a relativistic theory should modify Equation (1) to incorporate the relativistic effect in the dynamic of the system.

Ref [15] found that the Newtonian acceleration could be modified in such a way that it predicts correctly the perihelion precession of the planets. That modification was based on the application of two consecutive boosts of the time dilation and length contraction to the Newtonian concept of time and space interval.

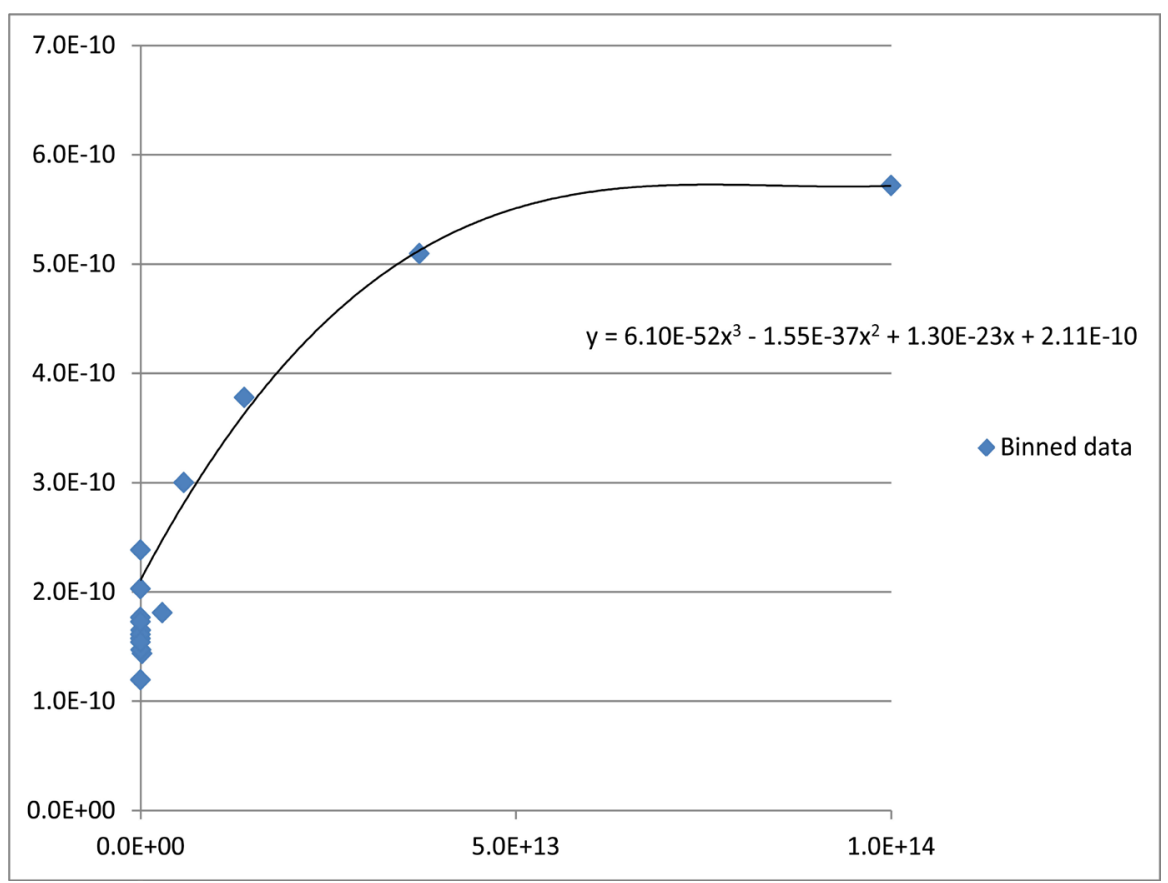

Figure 1. $a_{0}\left(\mathrm{~ms}^{-2}\right)$ vs. $M$ (Sun masses). Results of Equation (4) using the binned data and a cubic fit with preset intercept. 
The relativistic acceleration was expressed as

$$
a_{\gamma}=a\left(1-\beta^{2}\right)^{3}, \quad \beta=\frac{\mathrm{v}}{c}
$$

where

$a_{\gamma}$ : Relativistic acceleration.

$c$ : The speed of light in vacuum

Modifying Equation (1) according to Equation (8)

$$
F_{g}=\operatorname{ma} \mu\left(a, a_{0}\right)\left(1-\beta^{2}\right)^{3}
$$

Note that the interpolating function $\mu\left(a / a_{0}\right)$ was not modified (i.e. $a_{0}$ is also multiplied by $\left(1-\beta^{2}\right)^{3}$ in the denominator).

Equation 3(a) becomes $-\frac{1}{c^{6}} v^{10}+\frac{3}{c^{4}} v^{8}-\frac{3}{c^{2}} v^{6}+v^{4}-F r v^{2}-F r^{2} a_{0}=0$.

Equation 3(b) then becomes $-\frac{1}{c^{6}} v^{10}+\frac{3}{c^{4}} v^{8}-\frac{3}{c^{2}} v^{6}+v^{4}-G M \frac{v^{2}}{r}-G M a_{0}=0$

Therefore the baryonic Tully-Fisher relation is extended to relativistic speeds as

$$
-\frac{1}{c^{6}} v^{10}+\frac{3}{c^{4}} v^{8}-\frac{3}{c^{2}} v^{6}+v^{4}=G M a_{0}
$$

The dependence of $a_{0}$ on $M$ could be taken into account per the approximations to Equation (5). Note that Equation (10) can be derived from Equation (4) also and that it can be written in compact form as $\mathrm{v}^{4}\left(1-\beta^{2}\right)^{3}=G M a_{0}$. If the relativistic correction to $a_{0}$ is not considered then Equation (10) becomes $\mathrm{v}^{4}\left(1-\beta^{2}\right)^{6}=G M a_{0}$.

Table 2 shows the results of the speed calculation for very massive systems ranging from $10^{14}$ to $10^{21}$ in units of solar masses. Equation (10) was solved numerically just by evaluation of the function (evaluations were made every $1 \mathrm{~m} / \mathrm{s}$ ) in search for a sign change. There was not need to refine the search (once a sign change was found) because even though the evaluation to the left and right of

\begin{tabular}{|c|c|c|c|c|c|c|c|}
\hline \multirow{2}{*}{$\begin{array}{c}\mathrm{M} \\
(\text { solar } \mathrm{M})\end{array}$} & \multicolumn{6}{|c|}{$\mathrm{V}(\mathrm{km} / \mathrm{s})$} & \multirow[b]{2}{*}{ Equation (7) } \\
\hline & MOND & QI & НYВ & R-MOND & R-QI & R-HYB & \\
\hline $1.00 \mathrm{E}+14$ & $1.11 \mathrm{E}+03$ & $1.73 \mathrm{E}+03$ & $1.66 \mathrm{E}+03$ & $1.11 \mathrm{E}+03$ & $1.73 \mathrm{E}+03$ & $1.66 \mathrm{E}+03$ & $1.66 \mathrm{E}+03$ \\
\hline $1.00 \mathrm{E}+15$ & $1.98 \mathrm{E}+03$ & $3.07 \mathrm{E}+03$ & $2.95 \mathrm{E}+03$ & $1.98 \mathrm{E}+03$ & $3.07 \mathrm{E}+03$ & $2.95 \mathrm{E}+03$ & $2.99 \mathrm{E}+03$ \\
\hline $1.00 \mathrm{E}+16$ & $3.53 \mathrm{E}+03$ & $5.46 \mathrm{E}+03$ & $5.25 \mathrm{E}+03$ & $3.53 \mathrm{E}+03$ & $5.46 \mathrm{E}+03$ & $5.25 \mathrm{E}+03$ & $5.25 \mathrm{E}+03$ \\
\hline $1.00 \mathrm{E}+17$ & $6.27 \mathrm{E}+03$ & $9.71 \mathrm{E}+03$ & $9.33 \mathrm{E}+03$ & $6.27 \mathrm{E}+03$ & $9.72 \mathrm{E}+03$ & $9.34 \mathrm{E}+03$ & $9.22 \mathrm{E}+03$ \\
\hline $1.00 \mathrm{E}+18$ & $1.11 \mathrm{E}+04$ & $1.73 \mathrm{E}+04$ & $1.66 \mathrm{E}+04$ & $1.12 \mathrm{E}+04$ & $1.73 \mathrm{E}+04$ & $1.66 \mathrm{E}+04$ & $1.63 \mathrm{E}+04$ \\
\hline $1.00 \mathrm{E}+19$ & $1.98 \mathrm{E}+04$ & $3.07 \mathrm{E}+04$ & $2.95 \mathrm{E}+04$ & $1.99 \mathrm{E}+04$ & $3.10 \mathrm{E}+04$ & $2.97 \mathrm{E}+04$ & $2.88 \mathrm{E}+04$ \\
\hline $1.00 \mathrm{E}+20$ & $3.53 \mathrm{E}+04$ & $5.46 \mathrm{E}+04$ & $5.25 \mathrm{E}+04$ & $3.56 \mathrm{E}+04$ & $5.61 \mathrm{E}+04$ & $5.38 \mathrm{E}+04$ & $5.10 \mathrm{E}+04$ \\
\hline
\end{tabular}

Table 2. Circular speed calculation for very massive systems. QI (Quantized Inertia); HYB (mass dependent $a_{0}$ ). The prefix R stands for relativistic model. 
root resulted in a very large number, the estimated root (v) changed by very little amount (of the order of $0.01 \mathrm{~km} / \mathrm{s}$ ). More efficient root finding methods were not used because the CPU time was not an issue and to avoid problems with potential minimum, maximum, and inflection points.

Columns 2 - 4 show the results using the 3 ways of calculating $a_{0}$ per section 2 without relativistic consideration. The HYB model extrapolates $a_{0}$ linearly using the equation given by the point $\left(10^{14}, 5.72 \times 10^{-10}\right)$ in Table 1 and the point $\left(8.74 \times 10^{22}, 6.97 \times 10^{-10}\right)$ which approximate the current mass and acceleration of the universe. The results show the expected behavior of those models when extrapolated to very massive systems.

Columns 5 - 7 show the results of the 3 models considering the relativistic impact per equation 10 .

It is notified that for the last point in Table $2\left(M=10^{21}\right)$ an additional root was found (between $250 \times 10^{3}$ and $260 \times 10^{3} \mathrm{~km} / \mathrm{s}$ ) for R-QI and R-HYB models. It is believed that this is a result of the compiler/machine precision limits, noting that the search was stopped for all models at $0.9 c$ to avoid obtaining more than 10 roots for equation 10 which is mathematically impossible. Note that the values above correspond to $0.83 c$ and $0.87 c$ respectively.

The results of Table 2 show that equation 10 significantly impacts the circular speed (in magnitude of about $1000 \mathrm{~km} / \mathrm{s}$ or greater) only beyond about $10^{19}$ solar mass and that it results in an increase of the circular speed. That equation could then account for potential missing mass/energy in those massive systems assuming that those super massive structures could exist. Note however that the whole universe could be envisioned as a rotating cosmic structure (see some remarks in the next section).

The results of the extrapolation of Equation (7) to very massive systems, is shown in the last column of Table 2. It is remarkable that those results are fairly close to the results of the HYB model considering that Equation (7) does not 'know' anything about $a_{0}(M)$. The deviation from R-HYB at large values of mass is expected because Equation (7) was obtained based on a fit to the binned experimental data of significantly less mass where the relativistic impact is negligible.

\section{Additional Remarks}

-It is notified that a fitting to the data of Table 1 (adding the point $\left(M_{U}, \mathrm{CH}_{0}\right)$ and normalizing the mass such that $M_{U}=1$ ) with a single function was successfully performed after the completion of section 2 and 3; A non-linear regression with a function of the form $a_{0}(M)=-1.363626577 \times 10^{-5} M$

$+1.363680027 \times 10^{-5} \sqrt{M}+1.620133867 \times 10^{-10}$ was suggested by the computer application described in [19]. Note that the sum of the 3 coefficients is practically $c H_{0}$, that the independent term is close to MOND's $a_{0}$ and that $k \approx 4.3$ (see Equation (5)) which is not very far from $k \approx 5,8.5$ reported in [20] considering that the fitting in this work covers only a single binned data set which includes binned galaxy clusters. It could be worthy to apply the procedures of this 
work to other available data considering a comprehensive statistical assessment.

-The idea of a rotating universe has been proposed before by references dating back to at least the 1940's and experimental evidences have also been claimed previously (e.g. [13] [21] [22] and references therein).

\section{Summary and Concluding Remarks}

Based on an observed (using binned experimental data) strong correlation of $a_{0}$ with the mass of the galaxy clusters, it was proposed that $a_{0}$ should not be considered a constant, and that it could be represented as a function of the mass of the system. The results obtained in this way match better the binned experimental data when compared to the results of MOND and QI.

The relativistic impact was considered in super massive systems through the use of the extended Newtonian theory for gravitational bound systems. The impact resulted in an increase of the circular velocity of systems with mass beyond $\sim 10^{19}$ Msun.

It is remarked that the extension of the BTF relation as described here could contribute to explain the missing mass problem in galaxy clusters and other supermassive systems.

It could be worthy to compare the results of the HYB and R-HYB models to other experimental data.

\section{Acknowledgements}

The author thanks the anonymous reviewer for suggesting that the purpose and conclusions of the work should be added.

\section{References}

[1] Bertone, G. and Hooper, D. (2016) A History of Dark Matter. FERMILAB-PUB16-157-A. astro-ph.CO. arXiv:1605.04909v2

[2] Zwicky, F. (2008) The Redshift of Extragalactic Nebulae. Republication. Springer Science + Business Media LLC.

[3] Zwicky, F. (1937) On the Masses of Nebulae and Clusters of Nebulae. Astrophysical Journal, 86, 217. https://doi.org/10.1086/143864

[4] Tully, R.B. and Fisher, R.J. (1977) A New Method of Determining Distances to Galaxies. Astronomy \& Astrophysics, 54, 661-673.

[5] Milgrom, M. (1983) A Modification of the Newtonian Dynamics as a Possible Alternative to the Hidden Mass Hypothesis. Astrophysical Journal, 270, 365-370.

Milgrom, M. (1983) A Modification of the Newtonian Dynamics. Implications for Galaxies. Astrophysical Journal, 270, 371-389.

[6] Freeman, K.C. (1999) The Low Surface Brightness Universe. IAU Col. 171, ASP Conference Series, Vol. 170.

[7] McGaugh, S.S., et al. (2000) The Barionic Tully-Fisher Relation. arXiv:astro-ph/0003001v1

[8] Clowe, D., et al. (2006) A Direct Empirical Proof of the Existence of Dark Matter. arXiv:astro-ph/0608407v1

[9] Angus, G.W. and McGaugh, S.S. (2007) The Collision Velocity of the Bullet Cluster 
in Conventional and Modified Dynamics. Monthly Notices of the Royal Astronomical Society, 383, 417-423. arXiv:0704.0381v2[astro-ph]

[10] Nelson, A.H. (1988) On the Influence of Galaxy Magnetic Fields on the Rotation Curves in the Outer Discs of Galaxies. Monthly Notices of the Royal Astronomical Society, 233,115-121. https://doi.org/10.1093/mnras/233.1.115

[11] Brownstein, J.R. and Moffat, J.W. (2005) Galaxy Rotation Curves without Non-Baryonic Dark Matter. arXiv:astro-ph/0506370v4

[12] Mol, W.A. (2011) On the Gravitodynamics of Moving Bodies. Central European Journal of Physics, 9, 1151-1164. https://doi.org/10.2478/s11534-011-0030-7

[13] Zorba, S. (2012) Dark Energy and Dark Matter as Inertial Effects. arXiv:1210.3021[physics.gen-ph]

[14] McCulloch, M.E. (2012) Testing Quantized Inertia in Galactic Scales. arXiv1207.7007v1[physics.gen-ph]

[15] Quintero-Leyva, B. (2016) An Extended Newtonian Theory for Gravitational Bound Systems. Open Access Library Journal, 3, e2678. https://doi.org/10.4236/oalib.1102678

[16] Famey, B. and Binney, J. (2005) Modified Newtonian Dynamics in the Milky Way. Monthly Notices of the Royal Astronomical Society, 363, 603-608. https://doi.org/10.1111/j.1365-2966.2005.09474.x

[17] McGaugh, S.S., et al. (2010) The Baryon Content of Cosmic Structures. The Astrophysical Journal, 708, L14-L17. https://doi.org/10.1088/2041-8205/708/1/L14

[18] McGaugh, S.S. and Wolf, J. (2010) Local Group Dwarf Spheroidals: Correlated Deviations from the Baryonic Tully-Fisher Relation. The Astrophysical Journal, 722, 248-261. https://doi.org/10.1088/0004-637X/722/1/248

[19] (2017). http://www.xuru.org

[20] Quintero-Leyva, B. (2017) On the Characteristic Acceleration of MOND. Open Access Library Journal, 4, e3576.

[21] Berman, M.S. (2013) Realization of Einstein's Machian Program. arXiv:1302.2498v1[physics-gen-ph]

[22] Berman, M.S. and Gomide, F. (2013) Local and Global Stability of the Universe. Journal of Modern Physics, 4, 7-9. https://doi.org/10.4236/jmp.2013.48A002

Submit or recommend next manuscript to OALib Journal and we will provide best service for you:

- Publication frequency: Monthly

- 9 subject areas of science, technology and medicine

- Fair and rigorous peer-review system

- Fast publication process

- Article promotion in various social networking sites (LinkedIn, Facebook, Twitter, etc.)

- Maximum dissemination of your research work

Submit Your Paper Online: Click Here to Submit

Or Contact service@oalib.com 